\title{
Optical phase measurement emphasized
}

\section{Ertan Salik}

Ertan Salik, "Optical phase measurement emphasized," Proc. SPIE 9666, 11th Education and Training in Optics and Photonics Conference, 96661G (5 June 2009); doi: 10.1117/12.2207976

SDIE Event: Eleventh International Topical Meeting on Education and Training in Optics and Photonics, 2009, St. Asaph, United Kingdom 


\title{
Optical phase measurement emphasized
}

\author{
Ertan Salik \\ Physics Department, California State Polytechnic University, Pomona \\ 3801 W Temple Ave, Pomona, California 91768, USA \\ esalik@csupomona.edu
}

\begin{abstract}
In undergraduate optics laboratory, one thing that is not easily achieved is quantitative measurement of optical phase. The reason is that optical phase measurement usually requires expensive interferometers. We demonstrate measurement of relative optical phase shift upon total internal reflection. Total internal reflection, though known by every student of optics, is remembered by $100 \%$ reflection at an interface when angle of incidence is greater than the critical angle, that is, it seems all the same beyond the critical angle. This is not entirely true if one considers the optical phase, which keeps changing upon total internal reflection as the angle of incidence is varied. Furthermore, for linear polarization states perpendicular to or in the plane of incidence (sand $p$ - polarization), optical phase changes differently upon total internal reflection. Therefore, a linearly polarized beam composed of both s- and p-polarization undergoing total internal reflection becomes elliptically polarized. We show how to determine relative optical phase change between s- and p- polarization states through analysis of the outgoing elliptically polarized beam. Such optical phase change can also be theoretically calculated using Fresnel equations.
\end{abstract}

\section{INTRODUCTION}

For any harmonic signal, amplitude and phase information are both essential. For example, in communication applications, amplitude, frequency, and phase modulation schemes are all well established methods. In optical communications, however, intensity modulation is mostly used. Although phase modulation at optical frequencies is straightforward, it has never become a preferred method. The main reason for this is that direct phase detection at optical frequencies is extremely difficult, whereas intensity detection through photodiodes is straightforward. The difficulty for direct phase detection stems from the fact that one optical cycle is a few femtoseconds $\left(10^{-15}\right.$ second). Photodetectors that can respond in such a short amount of time have not yet been invented. Furthermore, in a typical optical communication system phase may constantly vary. Consider, for example, a semiconductor laser, a piece of optical fiber, and a photodetector. Unless the optical fiber is in an ultrastable condition, that is, neither temperature fluctuations nor vibrations occur in the environment, phase will constantly fluctuate due to temperature and stress induced changes in refractive index and length of the optical fiber. Consequently, detection of absolute phase at optical frequencies is impractical.

Although measurement of absolute optical phase is both very difficult and impractical, there are many optical devices that work based on optical phase changes, that is, relative optical phase. There are many interferometric devices that can measure relative optical phase through interference of two beams that are originally split after being emitted from a source. Among many types of optical sensors, those utilizing phase change as their working principle are usually superior to sensors based on optical intensity measurement.

In optics curriculum, numerous qualitative demonstrations of interference phenomena can be performed. Students, including those taking only introductory physics classes, can be easily shown the interference pattern through a double slit arrangement. Typically, a laser beam impinging on two narrow slits that are separated by a fraction of a millimeter results in an interference pattern on a screen. In optics laboratory classes, more advanced experiments, such as Michelson interferometer or Fabry-Perot interferometer are possible. In all these cases, most of the time, the student experience is limited to fringe counting, that is, more of a qualitative approach is utilized rather than a quantitative description of optical phase. 
An important phenomenon where optical phase manifests itself is total internal reflection. There are, for example, sensors dependent on relative phase shift upon reflection from an interface. ${ }^{1}$ Fresnel rhomb is an optical component that works based on optical phase shift upon total internal reflection. ${ }^{2,3}$ In typical experiments involving total internal reflection, students are asked to send a laser beam to a prism, and observe the reflected and refracted beams as the angle of incidence is varied. Of course, beyond the critical angle, one observes total internal reflection, which is simply $100 \%$ reflection, and the transmitted beam disappears. If the prism angles are known and measurement of the angle of incidence is carried out, then the critical angle can be determined. If the reflectance---ratio of the reflected to the incident optical power---at, for example, glass-air interface is measured, one can verify Fresnel equations. ${ }^{2,4-8}$ In all such experiments, however, one merely deals with optical power measurements, and optical phase changes are omitted. Students may leave the lab thinking that beyond the critical angle, total internal reflection is nothing but $100 \%$ reflection. This is not true. In fact, total internal reflection is a very interesting phenomenon with many subtle aspects. ${ }^{9}$ One of these subtleties is continuous change of optical phase upon total internal reflection. To study the optical phase change upon total internal reflection, one needs to perform a complete analysis using Fresnel equations.

Optical phase change can be measured interferometrically..$^{10}$ There are, however, multiple challenges that need to be faced in a typical interferometer setup. First, the setup needs to be on a sufficiently heavy optical table, and one needs to keep the arms of the interferometer stable. Such stringent conditions may make it difficult to emphasize quantitative characterization of optical phase in an undergraduate laboratory.

We propose in this paper a simple experiment emphasizing optical phase change based on measurement and analysis of elliptically polarized state of light that is formed upon total internal reflection. We present the relevant theory on elliptically polarized light and Fresnel equations, and demonstrate both qualitative and quantitative experiments that demonstrate optical phase shift upon total internal reflection.

\section{THEORY}

\subsection{Elliptically polarized light}

If electric field vector of an electromagnetic wave sweeps an ellipse, it is said to be elliptically polarized. For a plane wave traveling in the $z$ direction, and if $x$ and $y$ axes represent horizontal and vertical directions, respectively, the electric field vector of an elliptically polarized beam can be written as:

$$
\vec{E}=E_{0 x} \cos \left(k z-\omega t+\phi_{x}\right) \hat{i}+E_{0 y} \cos \left(k z-\omega t+\phi_{y}\right) \hat{j}
$$

Elliptical polarization is the most general expression of polarization state. Linear and circular polarization states are special cases. If $\phi_{x}=\phi_{y}$, we obtain linear polarization. If $\left|\phi_{x}-\phi_{y}\right|=\pi / 2$ and $E_{0 x}=E_{0 y}$ then we obtain circularly polarized light. More information on polarization can be found in any standard text in optics, such as ref. 2. Elliptically polarized light can be analyzed with various methods. ${ }^{11}$

\subsection{Fresnel equations and optical phase shift}

Fresnel equations result from Maxwell's equations solved at an interface. Reflectance depends on both the angle of incidence and the polarization state of the incident beam. A well-known effect illustrating the polarization dependence is of Brewster angle: For a light beam linearly polarized parallel to the plane of incidence---p-polarized or TM (transverse magnetic)---the reflected beam diminishes at a certain angle (Brewster angle or polarization angle); ${ }^{12}$ for a light beam linearly polarized perpendicular to the plane of incidence---s-polarized or TE (transverse electric)---there is always some reflection as the angle of incidence is varied. 
The reflection amplitude coefficients---ratio of reflected electric field to incident electric field---are needed to describe the amplitude and phase changes upon reflection at a dielectric interface. The reflection amplitude coefficients $r_{s}$ and $r_{p}$ for s- and p-polarized light, respectively, can be expressed as: ${ }^{2}$

$$
\begin{gathered}
r_{s}=\frac{\cos \theta_{i}-\sqrt{n_{t i}^{2}-\sin ^{2} \theta_{i}}}{\cos \theta_{i}+\sqrt{n_{t i}^{2}-\sin ^{2} \theta_{i}}} \\
r_{p}=\frac{n_{t i}^{2} \cos \theta_{i}-\sqrt{n_{t i}^{2}-\sin ^{2} \theta_{i}}}{n_{t i}^{2} \cos \theta_{i}+\sqrt{n_{t i}^{2}-\sin ^{2} \theta_{i}}}
\end{gathered}
$$

where $\theta_{i}$ is the angle of incidence, $n_{t i}=n_{t} / n_{i}$, and $n_{i}$ and $n_{t}$ are the refractive indices of the incident and the transmitted media, respectively. Using reflection amplitude coefficients, we can calculate reflectance by simply the product of an amplitude coefficient and its complex conjugate. Reflectance at air-glass interface for both external $\left(n_{i}<n_{t}\right)$ and internal $\left(n_{i}>n_{t}\right)$ reflection cases are shown in Fig. 1. Note that for internal reflection, the reflectance is constant at $100 \%$ beyond the critical angle.

As a light beam is reflected at a dielectric interface, not only does the reflectance depend on its state of polarization and the angle of incidence but also does the phase of the reflected beam. ${ }^{2,13-14}$ If $n_{i}<n_{t}$, reflection amplitude coefficients are real, and amplitude phase shift is either 0 or $\pi$. If, on the other hand, $n_{i}>n_{t}$, phase shift is 0 or $\pi$, or it varies continuously between 0 and $\pi$ in the case of total internal reflection, where reflection amplitude coefficients are complex. (Fig. 1) In order to calculate the phase shift upon reflection we can simply calculate the argument of the amplitude coefficients. The relative phase shift $\Delta \phi$ can be written as a function of $\theta_{i}$ and $n_{t i}$ as:

$$
\Delta \phi=\arg \left(r_{p}\right)-\arg \left(r_{s}\right)
$$

Fig. 2 shows the relative phase shift for total internal reflection at air-glass interface. This figure summarizes the goal of the experiments to be presented in this paper, that is, we will experimentally demonstrate the validity of the curve in Fig. 2, which shows that relative phase shift $\Delta \phi$ varies between 0 and about $45^{\circ}$ giving rise to elliptical polarization.

\subsection{Calculating transmission of an elliptically polarized beam through a linear polarizer}

A linear polarizer in Jones calculus ${ }^{15}$ can be described as follows:

$$
\mathcal{P}=\left(\begin{array}{cc}
\cos ^{2} \alpha & \cos \alpha \sin \alpha \\
\cos \alpha \sin \alpha & \sin ^{2} \alpha
\end{array}\right)
$$

where $\alpha$ is the angle between the horizontal (parallel to plane of incidence) and the polarization transmission axis. As shown in Fig. [exp1], if we send a linearly polarized beam $\vec{P}_{0}$ to the prism, then it becomes elliptically polarized $\left(\vec{P}_{1}\right)$ upon total internal reflection. We can express $\vec{P}_{0}$ and $\vec{P}_{1}$ as follows:

$$
\begin{gathered}
\vec{P}_{0}=\frac{1}{\sqrt{2}}\left(\begin{array}{l}
1 \\
1
\end{array}\right) \\
\vec{P}_{1}=\frac{1}{\sqrt{2}}\left(\begin{array}{c}
e^{i \Delta \phi} \\
1
\end{array}\right)
\end{gathered}
$$


If the elliptically polarized light $\vec{P}_{1}$, then, goes through a linear polarizer, the polarization vector out of the polarizer $\vec{P}_{2}$ can be obtained by applying the linear polarization matrix (Eq. 5) on $\vec{P}_{1}$ (Eq. 7):

$$
\vec{P}_{2}=\frac{1}{\sqrt{2}}\left(e^{i \Delta \phi} \cos \alpha+\sin \alpha\right)\left(\begin{array}{c}
\cos \alpha \\
\sin \alpha
\end{array}\right)
$$

If one measures the intensity through the polarizer as the polarization axis is turned between $\alpha=0^{\circ}$ and $\alpha=360^{\circ}$, the intensity of the transmitted beam will be given by:

$$
I(\alpha)=I_{\max } \vec{P}_{2}^{*} \cdot \vec{P}_{2}=\frac{I_{\max }}{2}[1+\sin (2 \alpha) \cos (\Delta \phi)]
$$

\section{EXPERIMENT}

\subsection{Qualitative demonstration of optical phase change upon total internal reflection}

Before any quantitative measurement, we can qualitatively show that there is indeed some optical phase change upon total internal reflection, which is revealed by modification of the polarization state. One can send a laser beam to a prism such that light is polarized at $45^{\circ}$ with the horizontal. If we place and rotate a polarizer in the path of the beam right before the beam enters into the prism, we can reduce transmission to almost zero, which occurs when polarization axis of the polarizer makes $90^{\circ}$ with the polarization direction of the beam. If we now take the polarizer-without modifying its polarization axis direction-and place it in the path of the beam after it exits the prism (Fig. 3), we notice that light is no longer totally blocked indicating modification of the polarization state. Rotating the polarizer axis will further reveal that the beam is not linearly polarized. Rather, it is now elliptically polarized.

Which event causes the polarization change? Entering the prism, exiting the prism, traveling in the prism, or total internal reflection? We will now discuss each of them and suggest simple experiments to investigate how each of these events can cause a polarization change.

As the beam enters and exits the prism it goes through glass-air interface, and it partially reflects. From Fig. 1, we know that reflectance and transmittance are both different functions of angle of incidence for $s-$ and $p$ polarizations. Therefore, if we send a beam that has equal s- and p-polarization components, then we might have some polarization change depending on the angle of incidence. We should also consider phase shifts. Except for total internal reflection we see from Fig. 1 that phase shift for reflection is 0 or 180 degrees. For transmitted beam there is no phase shift. ${ }^{2}$ Therefore, variation of reflectance based on polarization and phase shifts might cause a polarization change, but cannot result in elliptically polarization, but they can change the polarization direction of the linearly polarized light. For close to normal incidence, however, both polarizations have equal reflectance and transmittance for external and internal reflection cases, and consequently no polarization change is expected upon entry to and exit our of the prism.

For an ideal non-crystalline material, such as glass, one expects no birefringence. Therefore, it is unlikely that the polarization be modified while the beam is traveling in the prism. For crystals, such as calcite, depending on the polarization axis relative to the crystal axis, we can expect polarization modification. If there is any stress frozen-in during fabrication of the prism, however, then we can expect some stress-induced birefringence. We can check whether the prism has any stress-induced birefringence by sending a beam so that it goes through the prism without any total internal reflection. If we know the angles of incidence at each interface, then we can estimate the outgoing polarization, which should still be in a linear polarization state. If the polarization is not linear, we can conclude that the prism might have some stress-induced birefringence. 
If all the above tests show that there is no polarization modification that makes the outgoing polarization elliptically polarized, then the remaining option is that total internal reflection makes it happen, as expected from the Fresnel theory at interfaces. As displayed in Fig. 1, when light undergoes total internal reflection at glass-air interface, s- and p- polarization components will incur different phase shifts, which will result in elliptically polarized light.

\subsection{Determining phase shift difference by a polarizer and a photodetector}

Experimental setup is shown in Fig. 3. A single polarizer and a photodetector---with no quarter-wave plate--- can be enough to determine the relative phase shift. We used a silicon PIN photodiode (Thorlabs FDS100) with a load resistor, and read its voltage output with a digital voltmeter.

The best way to determine $\Delta \phi$ is to use nonlinear least squares fitting algorithm, which is readily available in commercial data analysis software, such as Igor Pro or Origin. After taking data for the transmitted intensity through the polarizer as a function of the angle $\alpha$, a nonlinear least squares fit to Eq. 9 with $\Delta \phi$ as a free parameter can be carried out. There is, however, a subtle point. First, $I_{\max }$ needs to be determined. Secondly, $\alpha=0$ corresponding to transmission axis being perfectly horizontal needs to be identified.

A better way to perform this nonlinear fit is to use two more free parameters: $I_{\max }$ and $\beta$, an angular offset for $\alpha$. Therefore, a nonlinear fit to:

$$
I(\alpha)=\frac{I_{\max }}{2}[1+\sin (2 \alpha+\beta) \cos (\Delta \phi)]
$$

where $I_{\max }, \beta$ and $\Delta \phi$ are free parameters is more appropriate.

Note that this method is only possible since we already know that the relative phase shift upon total internal reflection in the prism is less than $\pi / 2$. Otherwise, there will be some ambiguity in the measurement, as phase shift differences of $\Delta \phi$ and of $\Delta \phi+\pi$ result in the same transmitted intensity function. Fig. 4 shows data for $\theta_{i}=51.6^{\circ}$ fitted with this function revealing $\Delta \phi=45.7^{\circ} \pm 0.3^{\circ}$. We repeated the data collection and analysis for multiple angles of incidence thereby obtaining Fig. 5, which confirms excellent agreement between data and theoretical curve that was calculated using Fresnel theory.

\subsection{Total internal reflection in a right angle prism vs. other prism types}

No matter which method we use to determine the polarization state after the light beam comes out of the prism, we assume that the incoming polarization is always linear, and have equal components for each axis. In reality for the right angle prism shown in Fig. 1, this is strictly satisfied only when light enters and exits the prism along the normal---perpendicular to surface---, giving rise to an angle of incidence of of $45^{\circ}$ at the total-internalreflection surface. If the angle of incidence at entry is nonzero, however, then each polarization (parallel and perpendicular to the plane of incidence) will reflect differently making the polarization state of the transmitted beam change slightly.

An experimental solution to this problem is to use a half-cylinder prism, for which the beam always enters and exits along the normal, as shown in Fig.6. We know, however, that such special types of prisms are not readily available in most undergraduate laboratories, whereas $45^{\circ}-45^{\circ}-90^{\circ}$ prism is usually very common.

For a right-angle prism, we can adjust the initial polarization so that after the reflection at the first air-glass interface the desired polarization with equal components parallel and perpendicular to the plane of incidence is ensured. For the exit surface, the reflection needs to be accounted for in the theoretical curve, as the elliptically polarized light is reflected at the glass-air interface. Fortunately, this effect is not strong. We suggest the instructor to ask students to take data for total internal reflection with angles of incidence larger than $\approx 70^{\circ}$ at the glass--air interface, and ask them to explain the reason for slight discrepancy. 


\section{CONCLUSION}

We showed that one can teach about optical phase measurement utilizing a simple setup that measures relative optical phase shift upon total internal reflection. Fresnel equations can be used to calculate the relative phase shift. Because light that undergoes total internal reflection, analysis of elliptically polarized light enables us to determine the phase shift upon total internal reflection. One needs only a linear polarizer and a photodetector to quantitatively measure optical phase shift. In addition, within the experiment there are opportunities to use Jones calculus and nonlinear least squares fitting.

\section{ACKNOWLEDGEMENT}

We are very thankful to Michael Pantell for assistance in data collection. We are also extremely grateful to Prof. Peter Siegel for his comments and suggestions.

\section{REFERENCES}

[1] S. Patskovsky, M. Meunier, and A. V. Kabashin, "Phase-sensitive silicon-based total internal reflection sensor," Optics Express, Vol. 15, No. 19, 12523 (2007).

[2] E. Hecht, Optics (Addison-Wesley, San Francisco, CA, 2002), 4th. ed., 111--127.

[3] R. J. King, "Quarter-wave retardation systems based on the Fresnel rhomb principle," J. Sci. Instrum. 43, 617--622 (1966).

[4] R. K. P. Zia "Symmetric Fresnel Equations: An energy conservation approach," Am. J. Phys. 56, 555--558 (1988).

[5] A. S. Rupaal "Interpretation of Fresnel's Equations for Isotropic Dielectrics," Am. J. Phys. 34, 442--444 (1966).

[6] E. Collett “Mueller-Stokes Matrix Formulation of Fresnel's Equations," Am. J. Phys. 39, 517--528 (1971).

[7] W. T. Doyle "Graphical approach to Fresnel's equations for reflection and refraction," Am. J. Phys. 48, 643-647 (1980).

[8] W. T. Doyle "Scattering approach to Fresnel's equations and Brewster's law," Am. J. Phys. 53, 463--468 (1985).

[9] Whitehead, Lorne A; Mossman, Michele A, "Reflections on Total Internal Reflection," Optics and Photonics News, Vol. 20 Issue 2, pp.28-34 (2009).

[10] Kate M. Medicus, Marcus Chaney, John E. Brodziak, Jr., and Angela Davies, "Interferometric measurement of phase change on reflection," Applied Opt. Vol. 46, No. 11, pp. 2027-2035 (2007).

[11] S. Trester " On determining the phase difference introduced by a phase plate producing elliptically polarized light," Am. J. Phys. 61, 62--66 (1993).

[12] P. J. Ouseph, K. Driver, J. Conklin "Polarization of light by reflection and the Brewster angle," Am. J. Phys. 69, 1166--1168 (2001).

[13] C. S. Cook "Suggestion Regarding the Introduction of the Concept of Phase Change for Reflected Radiation," Am. J. Phys. 25, 92--94 (1957).

[14] G. Friedmann, H. S. Sandhu "Phase Change on Reflection from Isotropic Dielectrics," Am. J. Phys. 33, 135--138 (1965).

[15] R. C. Jones, "New calculus for the treatment of optical systems," J. Opt. Soc. Am. 31, 488--493 (1941). 


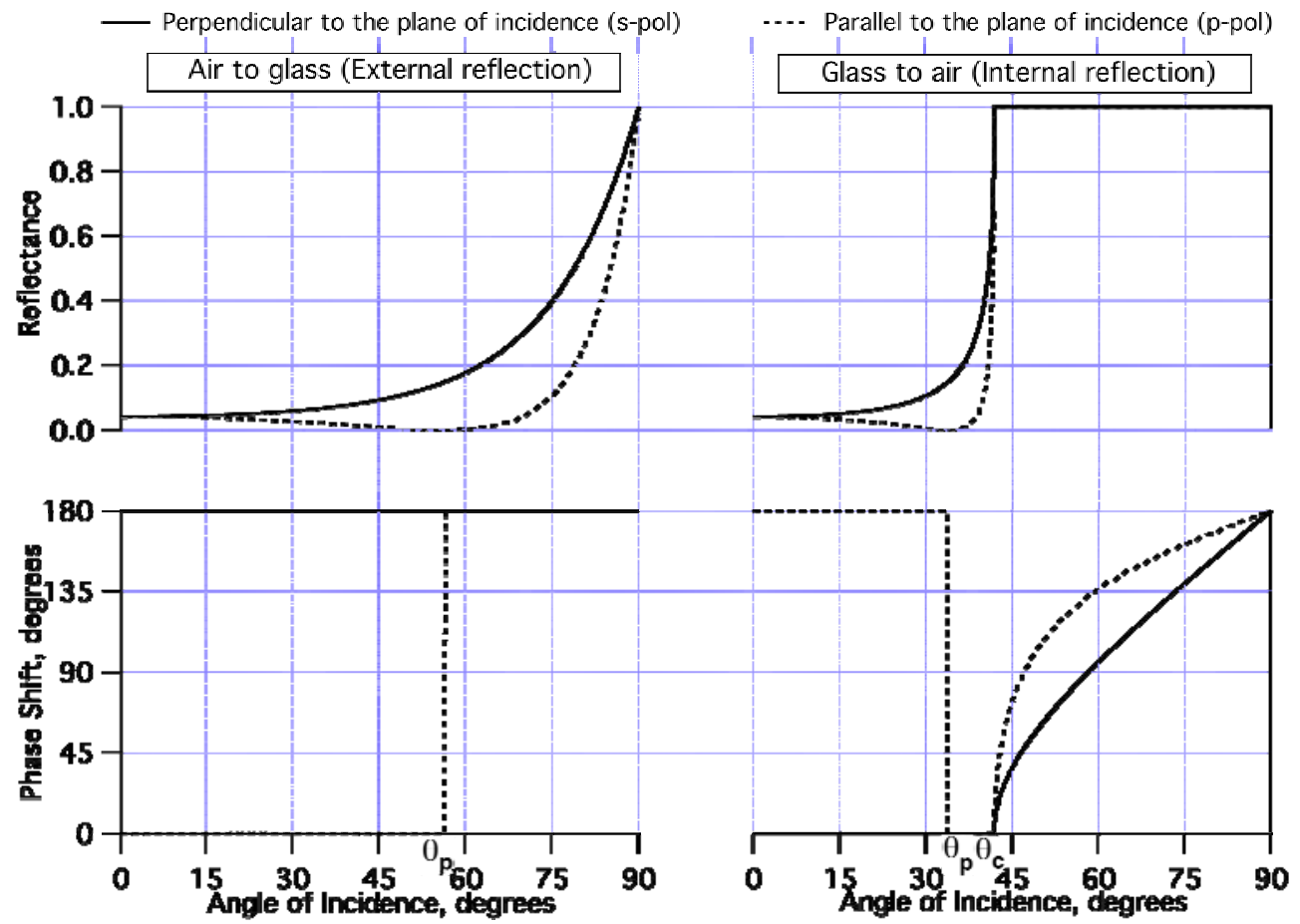

Fig. 1 Reflectance and phase shift upon reflection as a function of angle of incidence for s- and p-polarized beam at glass-air interface $\left(n_{\text {glass }}=1.5\right.$ and $\left.n_{\text {air }}=1.0\right)$. $\theta$ is the polarization angle (Brewster angle), and $\theta_{c}$ is the critical angle. When phase shift is constant $\left(0^{\circ}\right.$ or $\left.180^{\circ}\right)$ reflectance continuously varies for both external and internal reflection; when reflectance is constant at $100 \%$ (total internal reflection), then phase shift continuously varies.

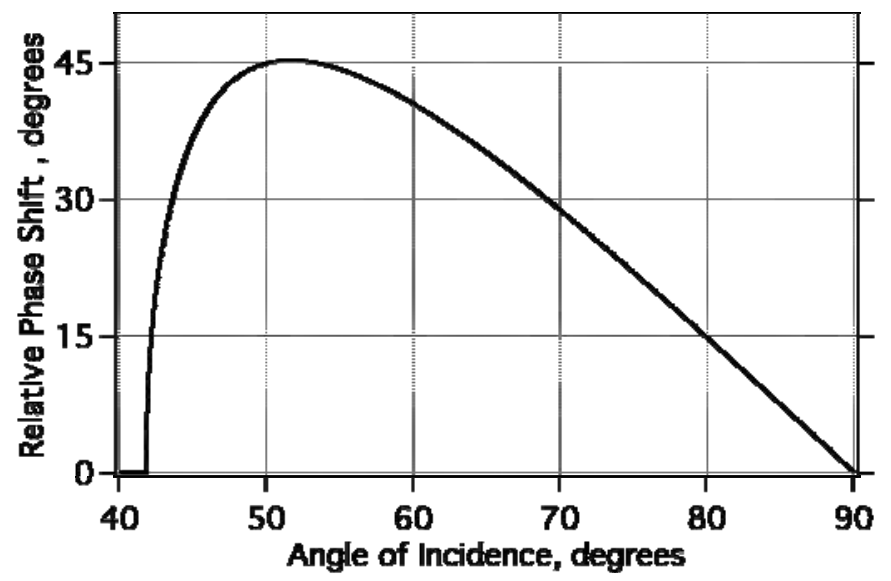

Fig. 2 Relative phase shift upon total internal reflection, which is the difference between phase shifts of s- and p-polarized light. The plot is produced for typical glass-air interface, where we used 1.00 and 1.50 for refractive indices of air and glass, respectively. 


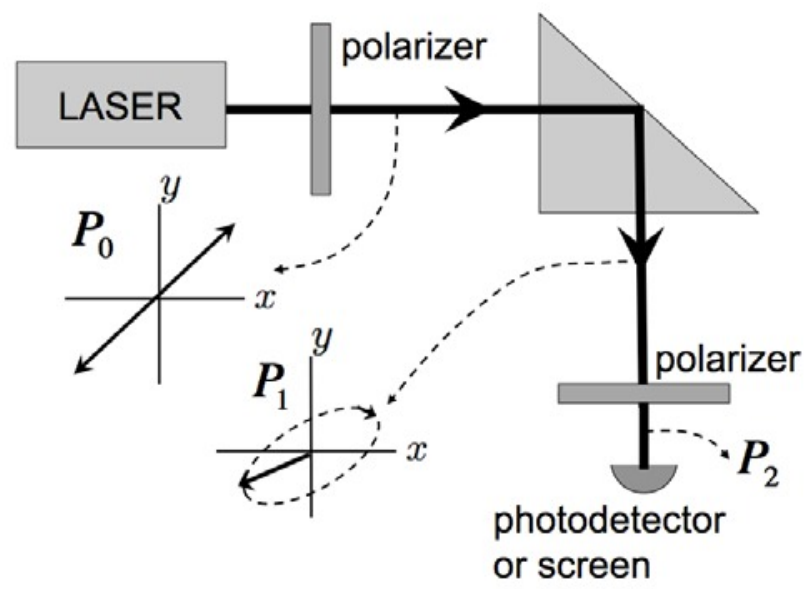

Fig. 3 Upon total internal reflection within the prism, light that is initially linearly polarized $\left(\mathrm{P}_{0}\right)$ becomes elliptically polarized $\left(P_{1}\right)$. By rotating the prism while keeping the plane of incidence unchanged, we can take data at various angles of incidence. With a polarizer, and a photodetector it is possible to determine the difference in optical phase change upon total internal reflection for s- and p- polarizations.

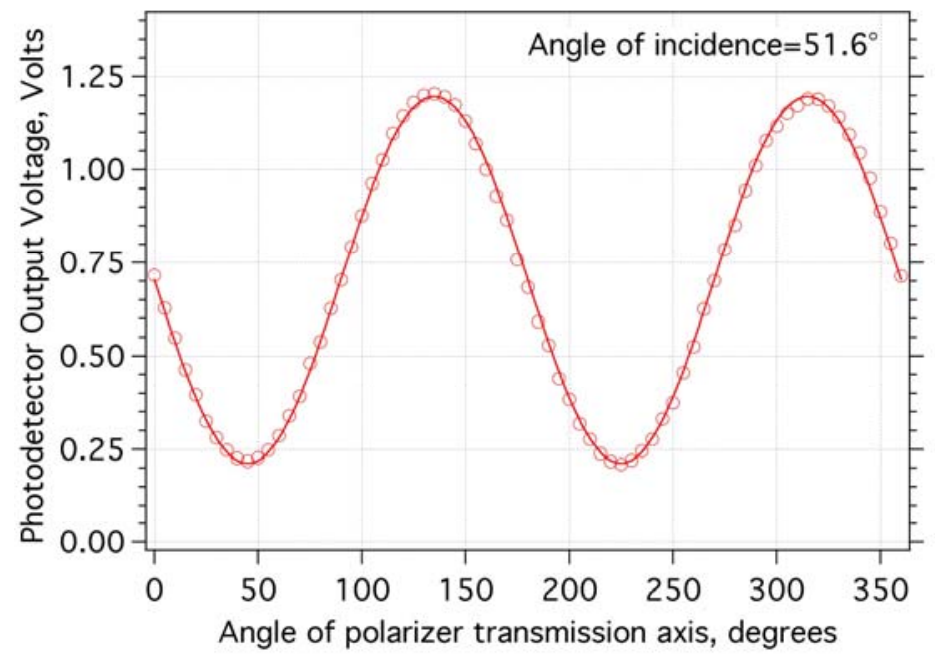

Fig. 4 Data for $\theta_{i}=51.6^{\circ}$ fitted to function in Eq. [fit]. From this nonlinear fit: $\Delta \phi=45.7^{\circ} \pm 0.3^{\circ}$. There are three free parameters for the fit: $I_{\max }, \beta$ and $\Delta \phi$. 


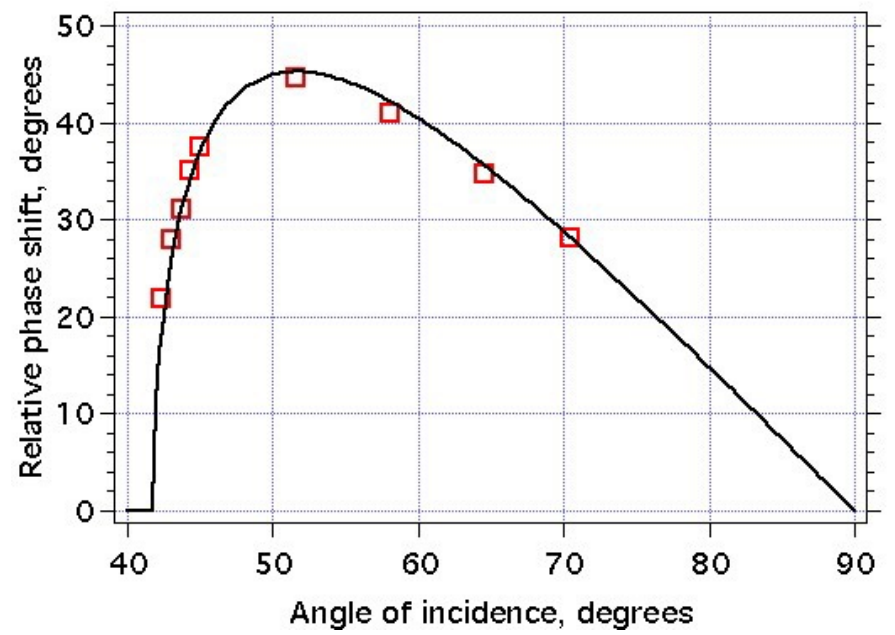

Fig. 5 Relative phase shift data at various angles of incidence. Theoretical curve is obtained for $n_{t i}=1.00 / 1.50$, that is, air-glass interface.

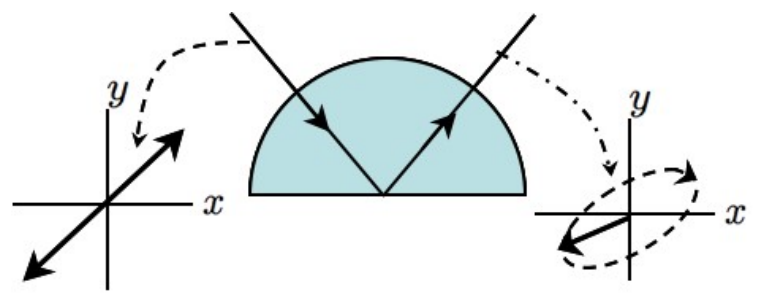

Fig. 6 Instead of using a right-angle prism, one can use a half-cylinder prism, for which the beam enters and exits along the normal if is directed towards the center. As a result, the polarization state will not be affected as the beam enters and exits the prism. 